\title{
Another route to a vaccine?
}

F. E. G. Cox

OVER the past few years, the search for a vaccine against malaria has shifted away from the infective sporozoite injected by the mosquito to the next phase of the life-cycle, the pre-erythrocytic stage in the liver (see box). A major problem, however, is how a protective mechanism against this stage might work. Now an important clue is reported on page 434 of this issue ${ }^{1}$, which stems from earlier observations by Adrian Hill and his colleagues that there is an association between the HLA class I antigen HLA-B53 and resistance to severe malaria ${ }^{2}$.

Their original hypothesis was that the HLA-B53 molecule might act as a binding site for a malaria antigen expressed on the surface of hepatocytes occupied by the parasites, and that this antigen might be a target for cytotoxic $\mathrm{T}$ lymphocytes (CTL). Using a series of 60 synthetic 8- 9- and 10-amino-acid peptides that matched the HLA-B53 molecule, and relating these to known sequences of sporozoite and liver-stage malaria antigens, Hill and his co-workers have pinpointed one likely candidate, a 9-amino-acid epitope, 1s6, from a specific liver-stage antigen LSA-1 (ref. 3). Interestingly, none of the sporozoite antigens is incriminated. The hope is that the 1 s 6 epitope could be a component of a vaccine that might induce a specific CTL response against the early stages in the liver and thereby confer some degree of immunity against the parasite. If hope turns into reality, this would be the first application of 'reverse immunogenetics' in the quest for epitopes suitable for vaccine design.

After many years of obscurity, the liver stage of the malaria life-cycle now seems to be a good target for immunological attack. The reasons for ignoring this stage until recently have been the excessive attention paid to antibodymediated responses to the sporozoite. In addition, there has been an unwillingness to accept the possibility that CTL could kill a parasite-infected cell, although it has been known for some time that the major defence against the cattle protozoa Theileria parva and $T$. annulata is not only CTL-dependent and restricted by the major histocompatibil- ity complex, but also that there are very effective vaccines based on the stimulation of this cytotoxic response ${ }^{4}$. Until now, however, the evidence for CTL involvement in malaria has been based largely on animal models and on sporozoite antigens either expressed during the early stages in the liver or left on the hepatocyte surface after invasion ${ }^{5}$. In humans, CTL responses to an immunogenic sporozoite antigen alone are not adequate to protect against malaria; furthermore, the protective epitopes contain polymorphic residues, suggesting that a protective immune response directed against one strain of the parasite might not be effective against another ${ }^{6}$. The advantage of the $1 \mathrm{~s} 6$ epitope is that it seems to be conserved, at least in the Gambia ${ }^{1}$, and is thus a potentially more useful molecule.

So far so good, but the significance of an association between HLA-B53 and protection against severe malaria has been questioned. The criteria used for defining severe malaria include cerebral involvement, hypoglycaemia and anaemia; the pathology of malaria may in part be due to a combination of immune responses ${ }^{7}$. Neither the proponents nor the opponents of the HLAB53 protective association hypothesis have considered the real problem, which
Life cycle of a malaria parasite showing the stages that might act as targets for vaccine-induced immunity. The cycle begins when infective stages, sporozoites (a), are injected directly into a blood vessel $(A)$ by a mosquito. The sporozoite is an obvious target for antibodymediated attack, but the success of vaccines against this stage has been disappointing. Sporozoites then enter liver hepatocytes $(B)$ where they divide to produce up to 10,000 merozoites. Possible targets include blocking sporozoite entry into the liver by antibodies (b), or killing early developmental stages in the liver by cell-mediated mechanisms (c) as described above. There is no evidence of any immune killing of the late-liver stage (d). Merozoites released into the bloodstream $(C)$ invade red blood cells $(e)$ where further division then occurs, resulting in the production of 8-16 merozoites which repeat the cycle. A number of antibodies inhibit invasion of red cells $(e)$ or the development of young $(f)$ or mature $(g)$ intraerythrocytic stages, but attempts to produce vaccines against these stages have met with mixed success. The bloodstream forms are also responsible for the pathology associated with malaria, part of which is immunologically mediated. Vaccines that neutralize toxic substances released by the parasites are being considered. Sexual stages $(h)$ are

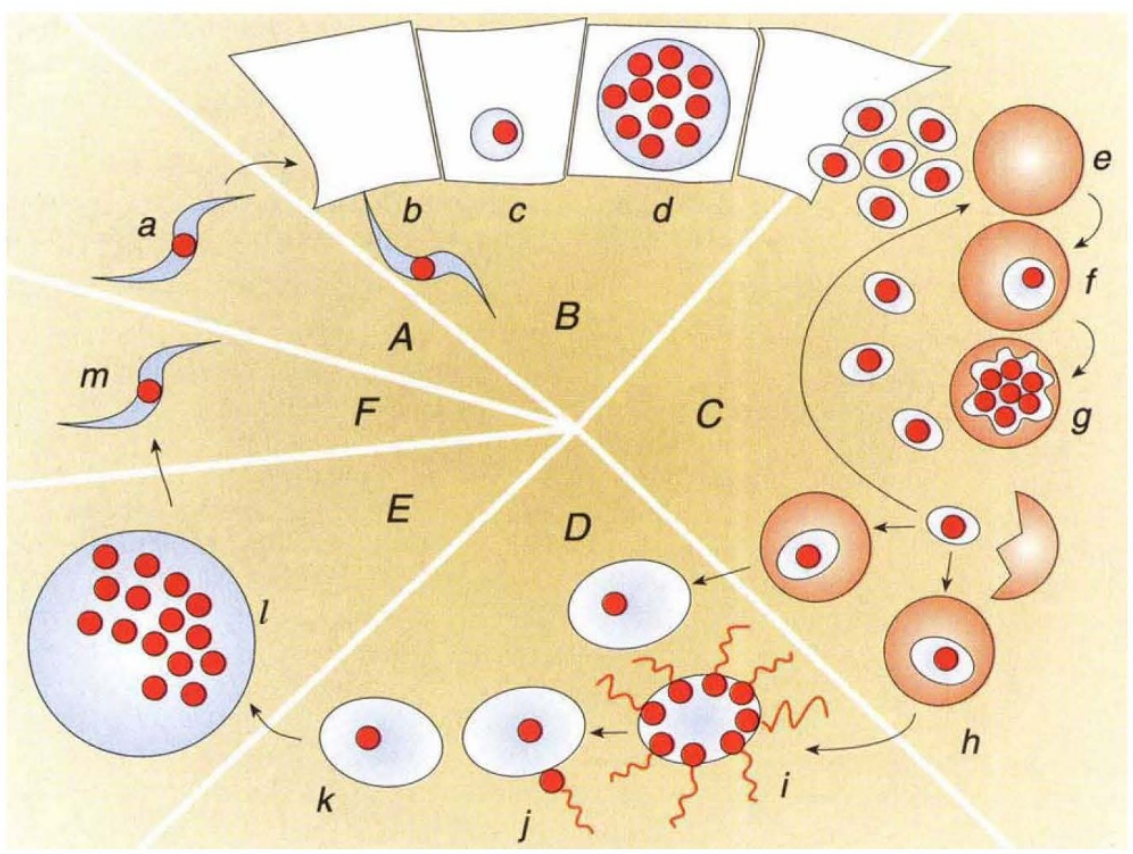

also produced in the blood and are taken up by a mosquito when it feeds $(D)$. Within the mosquito gut, a male gametocyte $(i)$ produces gametes that fertilize a female gamete $(j)$ to produce a zygote $(k)$. Antibodies, induced either naturally or artificially, inhibit all these sexual stages and vaccines against these stages have been used experimentally for blocking transmission. The zygote then forms an oocyst $(I)$ in the gut wall of the mosquito $(E)$, where another massive phase of multiplication results in the production of sporozoites $(m)$ that enter the salivary glands $(F)$ and are injected into the human host. There is no evidence of any immunity to these mosquito stages.
F. E. G. C. 